\title{
A Discontinuous Galerkin Method for the Shallow Water Equations in Spherical Triangular Coordinates
}

\author{
Matthias Läuter ${ }^{a 1}$, Francis X. Giraldo ${ }^{b}$, Dörthe Handorf ${ }^{a}$, Klaus Dethloff ${ }^{a}$ \\ a Alfred Wegener Institute for Polar and Marine Research in the Helmholtz Association, \\ P.O. Box 6001 49, 14401 Potsdam, Germany \\ b Naval Postgraduate School, Department of Applied Mathematics, Spanagel Hall 253A, \\ 833 Dyer Road, Monterey, CA 93943-5216, USA
}

\section{Abstract}

A global barotropic model of the atmosphere is presented governed by the shallow water equations and discretized by a Runge-Kutta discontinuous Galerkin method on an unstructured triangular grid. The shallow water equations on the sphere, a two-dimensional surface in $\mathbb{R}^{3}$, are locally represented in terms of spherical triangular coordinates, the appropriate local coordinate mappings on triangles. On every triangular grid element, this leads to a two-dimensional representation of tangential momentum and therefore only two discrete momentum equations.

The discontinuous Galerkin method consists of an integral formulation using a Rusanov numerical flux. A strong stability-preserving third order Runge-Kutta method is applied for the time discretization. The polynomial space of order $k$ on each curved triangle of the grid is characterized by a Lagrange basis and requires high-order quadature rules for the integration over elements and element faces. For the presented method no mass matrix inversion is necessary, exept in a preprocessing step.

The validation of the atmospheric model has been done considering steady-state and unsteady analytical solutions of the nonlinear shallow water equations. Experimental convergence was observed and the order of convergence $k+1$ was achieved. Furthermore, the article presents a numerical experiment, for which the third order time-integration method limits the model error. Thus, the time step $\Delta t$ is restricted by both, the CFL-condition and accuracy demands. As a second step of validation, the model could reproduce a known barotropic instability caused by a small initial perturbation of a geostrophic balanced jet stream. Conservation of mass was shown up to machine precision and energy conservation converges with decreasing grid resolution and increasing polynomial order $k$.

Keywords: Finite elements, Finite volumes, Shallow water equations, Triangular grid, Spherical geometry, Surface

\footnotetext{
${ }^{1}$ E-Mail: Matthias.Laeuter\{at\}awi.de (Matthias Läuter)

This work relates to Department of the Navy Grant N00014-07-1-4038 issued by the Office of Naval Research Global. The United States Government has a royalty-free licence throughout the world in all copyrightable material contained herein.

This article is submitted to Journal of Computational Physics
} 


\section{Introduction}

Modeling atmospheric flows for climate simulations as well as for weather prediction is a complex problem, due to the nonlinear structure of the dynamical and physical phenomena on widely varying spatial and temporal scales and their multi-scale interaction processes. Depending on the complexity of an atmospheric model the governing equations are the fundamental atmospheric conservation laws for mass, momentum and energy or appropriate simplifications of them. If the regarded equation set is a hyperbolic system, energetic shocks can develop theoretically. Although this is usually not the case in atmospheric models, the discretization should represent regions of scale collapse and breaking waves generating discontinuities in the velocity field; the discrete conservation properties of the DG method are appropriate for this task.

The shallow water equations (SWE), valid for a homogeneous atmosphere with small vertical velocities and horizontal velocities independent in the vertical direction, constitute a hyperbolic system of conservation laws. It is one of the simplest nonlinear hyperbolic systems, covering important planetary atmospheric features, like the Rossby wave formation.

For the spherical SWE the spatial domain is the sphere $S$, a two-dimensional surface in $\mathbb{R}^{3}$. In a regional or mesoscale SWE model the momentum is a twodimensional vector. In contrast, the Cartesian formulation of the spherical case in [8] represents the tangential momentum of the flow as a three-dimensional vector and includes a Lagrangian multiplier to constrain the momentum to be tangential. Applying this form to a numerical model usually leads to three momentum equations and requires a correction step to realize the constraint discretly, see e. g. [13]. Models in standard spherical coordinates realize a two-dimensional momentum representation but have to pay additional attention to phenomena near the poles due to singularities of the coordinate mapping, see e. g. [18].

The idea to avoid the drawbacks of the Cartesian and the spherical coordinates formulation is to represent the spherical SWE in terms of local coordinate transformations. On a cubed-sphere grid, a spherical quadrilateral grid, [20] and [21] achieved a two-dimensional momentum representation avoiding any pole problem. The presented model provides these properties on spherical unstructured triangular grids using spherical triangular coordinates. Using coordinate independent differential operators on $S$ known from differential geometry, the spherical SWE are considered in flux form on the surface $S$. Spherical triangular coordinates, that is appropriate local coordinate mappings $\gamma_{E}$ on a curved triangle $E$, yield the twodimensional representation of tangential momentum vectors. Numerous numerical methods have been proposed for future global atmospheric models including finite volumes [18], [21], spectral elements [25], [10], and DG methods. We have selected the DG method for our model because it allows us to achieve high-order accuracy as in spectral elements while conserving all quantities both locally and globally as in finite volumes. Furthermore, our use of unstructured triangular grids allows for much flexibility in future work on adaptivity.

DG methods have been already successfully applied to the spherical SWE in [12], [20] and [11]. DG methods in combination with Runge-Kutta (RK) time discretiza- 
tions, see the review in [5], can be characterized as a high-order generalization of finite volume methods. This method is robust, high-order accurate, locally conservative with high potential for parallelization, see [11].

The organization of this article is as follows. In Sec. 2 the governing spherical SWE, are given using surface differential operators. Section 3 describes the numerical discretization by a RK-DG method applying spherical triangular coordinates. In Sec. 4 the barotropic model of the atmosphere based on the discretization is validated in terms of steady-state and unsteady analytical solutions and a barotropic instability generated by a small initial perturbation.

\section{Spherical Shallow Water Equations}

The spherical shallow water equations (SWE) are a system of conservation laws for the geopotential layer depth (mass) and the flow momentum. Because the integration domain of the SWE is the sphere, a two-dimensional surface in $\mathbb{R}^{3}$, the system can be formulated in the surrounding Cartesian space $\mathbb{R}^{3}$, see [8]. Côté's formulation is equivalent to a conservative form of the SWE on the surface $S$, which is the formulation used further below.

Let us consider the sphere $S=\{x \in \mathbb{R}|| x \mid=a\}$ with the Earth's radius $a=$ $6.371 \times 10^{6} \mathrm{~m}$, the geopotential layer depth $\Phi: S \times \mathbb{R}^{+} \rightarrow \mathbb{R}$, the tangential velocity field $u: S \times \mathbb{R}^{+} \rightarrow \mathbb{R}^{3}$ with $u(x, t) \in T_{x}(S)$ and the conserved variable $q=\left(\Phi, \Phi u^{\mathrm{T}}\right)^{\mathrm{T}}$. Then, the SWE in conservative form on the surface $S$ are

$$
\partial_{t} q+\operatorname{div}_{S} f(q)=F(x, q) \quad \text { in } S \times \mathbb{R}^{+} .
$$

Thereby, the flux function and the right hand side are

$$
\begin{gathered}
f(q)=\left(\begin{array}{c}
f_{\Phi}(q) \\
f_{U}(q)
\end{array}\right), \quad f_{\Phi}(q)=\Phi u, \quad f_{U}(q)=\Phi u \otimes u+\frac{\Phi^{2}}{2} \operatorname{Id}_{3}, \\
F(x, q)=\left(\begin{array}{c}
0 \\
F_{U}(x, q)
\end{array}\right), \quad F_{U}(x, q)=-f_{c} \Phi k \times u-\Phi \nabla_{S} \Phi_{B}-\frac{\Phi u^{2}}{a} k
\end{gathered}
$$

with the Earth's angular velocity $\Omega=7.292 \times 10^{-5} \mathrm{~s}^{-1}$, the space dependent Coriolis parameter $f_{c}(x)=2 \Omega \frac{x \cdot e_{3}}{|x|}$, the normal unit vector $k(x)=\frac{x}{|x|}$ outward on $S$ and the identity mapping $\operatorname{Id}_{3}$ in $\mathbb{R}^{3}$. See the appendix for the definition of the differential operators on $S$. Three prognostic equations for the momentum $\Phi u$ appear in (1), whereas the momentum is forced to be tangential on $S$ by the Lagrangian multiplier $-\frac{\Phi u^{2}}{a} k$. Due to this forcing term no global conservation of momentum can be expected, which is in contrast to the two-dimensional shallow water equations. As a consequence of (1) mass is locally globally conserved while energy is only globally conserved.

\section{Discontinuous Galerkin Method}

The Discontinuous Galerkin (DG) method is applied to the conservative form (1) of the SWE on the surface $S$. Based on a given triangulation on every curved triangle 
(element) $E$ spherical triangular coordinates, which are local coordinate mappings $\gamma_{E}$ on each $E$, are introduced. Using $\gamma_{E}$ polynomial spaces of high-order are defined on each curved element. The polynomial representation on each grid element uses high-order Lagrange polynomials with respect to Fekete points. This approach leads to the local representation of the tangential momentum fields by two components only. An integral form of (1) leads to the space-discrete DG method including a Rusanov numerical flux. For this method high-order quadrature rules are applied and, except in a preprocessing step, no mass matrix inversion has to be evaluated. Finally the semi-discrete problem is solved by a strong stability-preserving explicit Runge-Kutta (RK) method. The fully discrete RK-DG method avoids any kind of explicit smoothing such as diffusion or filter operators.

\subsection{Spherical Triangular Coordinates}

Let $E \subset S$ be a relative open spherical triangle bounded by great circles and defined by its vertices $x_{0}, x_{1}, x_{2} \in S$. Then we define for $E$ the local coordinate mapping $\gamma_{E}$, or the spherical triangular coordinates, by

$$
\gamma_{E}: D \rightarrow E, \quad \gamma_{E}(y)=a \frac{x_{p}(y)}{\left|x_{p}(y)\right|}
$$

Here, $D=\left\{y \in \mathbb{R}^{2} \mid 0<y_{1}, y_{2} ; y_{1}+y_{2}<1\right\}$ is a two dimensional reference triangle, and $x_{p}(y)=x_{0}+y_{1}\left(x_{1}-x_{0}\right)+y_{2}\left(x_{2}-x_{0}\right)$ an auxiliary planar mapping. Following the notation in the appendix, a basis of $T_{x}(S)$, Gram's determinant and the Christoffel symbols are given with $i, j, k=1,2$

$$
\begin{gathered}
b_{i}=\frac{a}{\left|x_{p}\right|}\left(\left(x_{i}-x_{0}\right)-\left(x_{i}-x_{0}\right) \cdot \frac{x_{p}}{\left|x_{p}\right|} \frac{x_{p}}{\left|x_{p}\right|}\right), \\
g=\left(b_{1} \times b_{2}\right)^{2}, \quad \Gamma_{j k}^{i}=-\frac{x_{p}}{x_{p}^{2}} \cdot\left(\left(x_{j}-x_{0}\right) \delta_{k}^{i}+\left(x_{k}-x_{0}\right) \delta_{j}^{i}\right) .
\end{gathered}
$$

\subsection{Discrete Function Space}

Let $\mathcal{T}=\{E \subset S \mid E$ spherical triangle, $E$ open in $S\}$ be a finite conformal triangulation of the sphere - that is, a triangular grid without hanging nodes. For the presented model, $\mathcal{T}$ is constructed by the grid generator AMATOS, see [1]. Dependent on the grid level $l$, an icosahedral coarse grid $\mathcal{T}_{0}$ is refined in $l$ steps, in which every triangle of $\mathcal{T}_{0}$ is divided by bisection. This leads to an unstructured spherical triangulation $\mathcal{T}$ with nearly uniform grid resolution, see figure 1.

The polynomial space of the polynomials of degree at most $k \geq 0$ on every element $E \in \mathcal{T}$ is defined by

$$
P^{k}(E)=\left\{\varphi: E \rightarrow \mathbb{R} \mid \varphi \circ \gamma_{E} \in P^{k}(D)\right\}
$$

where $\gamma_{E}$ is the spherical triangular coordinate mapping (2). Thus, the coordinate mapping $\gamma_{E}$ defines as well the curved geometry of $E$ as the polynomial space on $E$. This technique for curved elements is similar to the definition of isoparametric finite elements, see [3], but with an analytically non-polynomial mapping $\gamma_{E}$. 

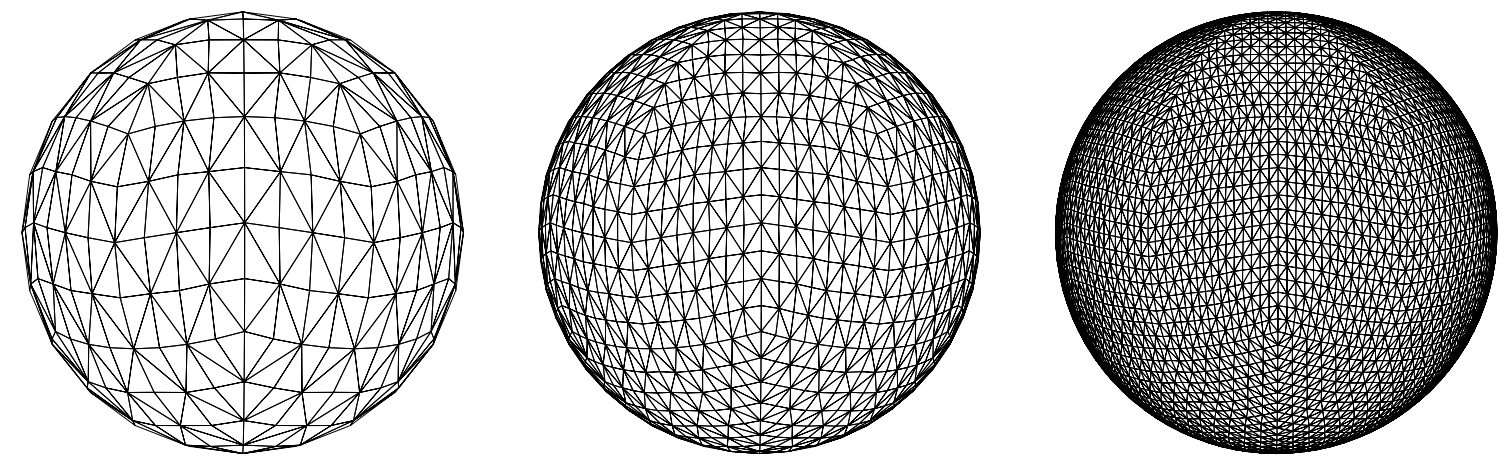

Figure 1: Section 3.2, Uniform grid, Grid resolution 2058km (left), 1041km (middle), $522 \mathrm{~km}$ (right).

Every polynomial $p \in P^{k}(E)$ is represented by a multivariate Lagrange basis $\left(\varphi_{i}\right)_{i=1, \ldots, N_{k}}$ of $P^{k}(E)$, with $N_{k}=\frac{(k+1)(k+2)}{2}$, associated with the Lagrange points $\left(x_{i}\right)_{i=1, . ., N_{k}}$, that is for $x \in E$

$$
p(x)=\sum_{i=1}^{N_{k}} \varphi_{i}(x) p\left(x_{i}\right) .
$$

Thereby, $\left(x_{i}\right)_{i=1, . ., N_{k}}$ as well as $\left(\varphi_{i}\right)_{i=1, . ., N_{k}}$ are defined by $\varphi_{i}=\tilde{\varphi}_{i} \circ \gamma_{E}^{-1}$ and $x_{i}=$ $\gamma_{E}\left(\tilde{x}_{i}\right) .\left(\tilde{\varphi}_{i}\right)_{i=1, . ., N_{k}}$ is the multivariate Lagrange basis of $P^{k}(D)$ associated with the Fekete points $\left(\tilde{x}_{i}\right)_{i=1, . ., N_{k}}$ in $D$ derived from the electrostatics principle, see [15].

Based on the polynomial space $P^{k}(E)$, the discrete discontinuous function spaces for the scalar fields and tangential vector fields are defined by

$$
\begin{aligned}
& V_{\Phi}=\left\{\Phi \in L^{\infty}(S)|\forall E \in \mathcal{T}: \Phi|_{E} \in P^{k}(E)\right\}, \\
& V_{U}=\left\{U \in L^{\infty}\left(S, \mathbb{R}^{3}\right)|\forall E \in \mathcal{T}: U|_{E}=U^{1} b_{1}+U^{2} b_{2} \text { with } U^{1}, U^{2} \in P^{k}(E)\right\}, \\
& V_{U}^{*}=\left\{U \in L^{\infty}\left(S, \mathbb{R}^{3}\right)|\forall E \in \mathcal{T}: U|_{E}=U_{1} b^{1}+U_{2} b^{2} \text { with } U_{1}, U_{2} \in P^{k}(E)\right\} .
\end{aligned}
$$

Because $\Phi \in V_{\Phi}, U \in V_{U}, V \in V_{U}^{*}$ are polynomials on each grid element $E$, the condition $\Phi, U, V \in L^{\infty}$ does not constitute an additional constraint to the discrete functions. The function spaces $V_{U}$ and $V_{U}^{*}$ contain tangential vector fields, that is $U(x) \in T_{x}(S)$ for $U \in V_{U} \cup V_{U}^{*}$. For every momentum $U \in V_{U}$ the restriction $\left.U\right|_{E}$ is a vector field having polynomial components with respect to $b_{1}$ and $b_{2}$. Test functions for the momentum equation are to be vector fields $U \in V_{U}^{*}$ where their restriction $\left.U\right|_{E}$ has polynomial components with respect to the dual basis $b^{1}$ and $b^{2}$.

Remark 1 The discrete function spaces $V_{U}$ and $V_{U}^{*}$ for the tangential vector fields incorporating spherical triangular coordinates ensure the two-dimensional representation of the momentum in (1). This denotes a reduction compared to the threedimensional representation in the Cartesian coordinate system used in [11] and [13]. Further, this approach avoids any kind of projection step incorporating a discrete version of the Lagrangian multiplier in the numerical scheme. 


\subsection{Space discrete formulation}

The starting point for the space discrete formulation is an approriate integral form of the conservation law. This is obtained multiplying (1) with a smooth (continuous in $S$ with derivatives) test function $p=(\varphi, V)^{\mathrm{T}}$, assuming a smooth solution $q$ of (1), integrating over $E \in \mathcal{T}$ and applying (10), that is

$$
\int_{E}\left(p \cdot \partial_{t} q-f(q): \nabla_{S} p\right) d x+\int_{\partial E} p \cdot f(q) \cdot \nu_{E} d \sigma=\int_{E} p \cdot F(x, q) d x .
$$

Here $f(q): \nabla_{S} p=f_{\Phi}(q) \cdot \nabla_{S} \varphi+\sum_{i=1}^{3} e^{i} \cdot f_{U}(q) \cdot \nabla_{S}\left(V \cdot e_{i}\right)$ and $\nu_{E}$ is the normal unit vector outward on $\partial E$. This integral form of (1) is to be the condition that the space-discrete solution $q_{h}(t) \in V_{\Phi} \times V_{U}$ has to fulfill, that is

$$
\begin{gathered}
\forall p \in V_{\Phi} \times V_{U}^{*}, \quad \forall E \in \mathcal{T} \\
\int_{E}\left(p \cdot \partial_{t} q_{h}-f\left(q_{h}\right): \nabla_{S} p\right) d x+\int_{\partial E} p^{i n} \cdot h_{E}\left(x, q_{h}^{i n}, q_{h}^{\text {out }}\right) d \sigma=\int_{E} p \cdot F\left(x, q_{h}\right) d x .
\end{gathered}
$$

By means of the discrete equation, $q_{h}$ as well as the test functions $p$ are in discrete function spaces. The function space $V_{U}^{*}$ is used for $V$ instead of $V_{U}$ to simplify the discrete representation (6) of (4). Further, due to the discontinuities of $q_{h}=$ $(\Phi, U)=(\Phi, \Phi u)$ along the edges of the triangles, the values of the flux function $f\left(q_{h}\right)$ are not defined on the boundaries $\partial E$. That is why, in the boundary integral of (4) the flux $f\left(q_{h}\right)$ is replaced by the Rusanov numerical flux

$$
h_{E}\left(x, q_{h}^{\text {in }}, q_{h}^{\text {out }}\right)=\frac{1}{2}\left[\left(f\left(q_{h}^{\text {in }}\right)+f\left(q_{h}^{\text {out }}\right)\right) \cdot \nu_{E}(x)-\lambda\left(q_{h}^{\text {out }}-q_{h}^{\text {in }}\right)\right],
$$

with the maximum wave speed $\lambda=\max \left(\left|u^{\text {in }} \cdot \nu_{E}\right|+\sqrt{\Phi^{\text {in }}},\left|u^{\text {out }} \cdot \nu_{E}\right|+\sqrt{\Phi^{\text {out }}}\right)$ in system (1). Below the notation $h_{E}=\left(h_{\Phi}, h_{U}\right)^{\mathrm{T}}$ regarding the scalar and momentum components will be used.

To obtain a matrix formulation of (4), the decomposition $q_{h}=(\Phi, U)$ with $\Phi \in V_{\Phi}$ and $U \in V_{U}$ is regarded. Using the decomposition (3) with regards to the Lagrange basis in $E \in \mathcal{T}$ yields

$$
\Phi(x, t)=\sum_{i=1}^{N_{k}} \Phi_{i}(t) \varphi_{i}(x), \quad U(x, t)=\sum_{i=1}^{N_{k}} \sum_{l=1}^{2} U_{i}^{l}(t) \varphi_{i}(x) b_{l}(x),
$$

the representation of $q_{h}$ in $E$ in terms of its component vector

$$
\hat{q}_{h, E}=\left(\Phi_{1}, . ., \Phi_{N_{k}}, U_{1}^{1}, . ., U_{N_{k}}^{1}, U_{1}^{2}, . ., U_{N_{k}}^{2}\right), \quad \hat{q}_{h}=\left(\hat{q}_{h, E}\right)_{E \in \mathcal{T}} .
$$

As expected, the tangential momentum field $U$ in $E$ is represented by the last $2 N_{k}$ components of $\hat{q}_{h, E}$ only. Then, $q_{h}$ is a solution of (4) if and only if in every $E \in \mathcal{T}$ for all $j=1, . ., N_{k}$ and $l=1,2$ the following equations hold

$$
\begin{aligned}
\sum_{i=1}^{N_{k}} \partial_{t} \Phi_{i} \int_{E} \varphi_{j} \varphi_{i} d x-\int_{E} f_{\Phi}\left(q_{h}\right) \cdot \nabla_{S} \varphi_{j} d x+\int_{\partial E} \varphi_{j} h_{\Phi} d \sigma & =0 \\
\sum_{i=1}^{N_{k}} \partial_{t} U_{i}^{l} \int_{E} \varphi_{j} \varphi_{i} d x-\int_{E} f_{U}^{l} \cdot \nabla_{S} \varphi_{j} d x+\int_{\partial E} \varphi_{j} h_{U}^{l} d \sigma & =\int_{E} \varphi_{j} \tilde{F}_{U}^{l} d x .
\end{aligned}
$$


Here the notation $f_{U}^{l}=b^{l} \cdot f_{U}\left(q_{h}\right) h_{U}^{l}=b^{l} \cdot h_{U}, \tilde{F}_{U}^{l}=b^{l} \cdot F_{U}-\sum_{i, m=1}^{2} f_{U}^{i m} \Gamma_{m i}^{l}$, $F_{U}^{l}=b^{l} \cdot F_{U}, f_{U}^{i m}=b^{i} \cdot f_{U}\left(q_{h}\right) \cdot b^{m}$ has been used. Using the dual momentum space $V_{U}^{*}$ for the test functions in (4) leads to the desirable separation of equations for the momentum components for $\left(U_{i}^{1}\right)_{i=1, . ., N_{k}}$ and $\left(U_{i}^{2}\right)_{i=1, . ., N_{k}}$ in (6). Eq. (6), for $l=1,2$, are two space discrete momentum equations, only.

To proceed with the space discrete system further below Eqs. (5) and (6) are written in the compact form

$$
\frac{\partial q_{h}}{\partial t}=L\left(q_{h}\right)
$$

with an appropriate right hand side operator $L$. The evaluation of $L\left(q_{h}\right)$ includes the evaluation of integrals over $E$ and $\partial E$ using the representation formulae (11). The integrands include as well the flux function $f$ in (1) as the surface geometry represented by Gram's determinant $g$. In each triangle $E$ quadrature rules of order $2 k$ are applied given in [24], [7], [19], [6]. On each edge of $E$ standard Gauss-Lobatto rules of order $2 k-1$ are applied, since Fekete points are in fact Gauss-Lobatto points along the edges, see [2]. Although [5] indicates, that quadrature rules of order $2 k+1$ along the edges are to be used for $k+1$-order formal accuracy, this would slow down the scheme. Experiments with a strong DG method in [11] have shown satisfying results applying $2 k-1$ order quadrature. Furthermore, we rely on the superconvergence property of outflow flux integrals analyzed in [16] to motivate our use of $2 k-1$ quadrature for the boundary integrals.

Remark $2 h_{E}\left(x, q_{h}^{\text {in }}, q_{h}^{\text {out }}\right)$ is a function of space and the conserved variables only, that is $h_{E}$ is independent on the local coordinate mapping $\gamma_{E}$. On the other hand, the formulations (5) and (6) depend on the coordinate mapping $\gamma_{E}$ because these determine the components $\Phi_{i}$ and $U_{i}^{l}$ regarding the polynomial basis $\left(\varphi_{i}\right)_{1, . ., N_{k}}$ of $P^{k}(E)$.

Remark 3 If the triangulation $\mathcal{T}$ is time independent, the mass matrix entries $M_{i j}=\int_{E} \varphi_{j} \varphi_{i} d x$ can be pre-evaluated once. Thus, multiplying (5) and (6) by $M^{-1}$ leads to the substitution of $\varphi_{j}$ and $\nabla_{S} \varphi_{j}$ by $M_{i j}^{-1} \varphi_{j}$ and $M_{i j}^{-1} \nabla_{S} \varphi_{j}$, respectively. The resulting equations allow the evaluation of $\partial_{t} \hat{q}_{h}$ avoiding any runtime mass matrix inversion.

\subsection{Runge-Kutta Method}

A strong stability-preserving (SSP) explicit third-order Runge-Kutta (RK) method, see [14], is used to solve the ordinary differential equation (7), that is for every time step $t^{n} \rightarrow t^{n+1}$

$$
\begin{aligned}
q^{(0)} & =q_{h}^{n}, \quad q^{(i)}=q^{(0)}+\tau \sum_{j=0}^{i-1} c_{i j} L\left(q^{(j)}\right), \quad i=1, \ldots, s \\
q_{h}^{n+1} & =q^{(s)},
\end{aligned}
$$

where $s=3$,

$$
\left(c_{10}, c_{20}, c_{21}, c_{30}, c_{31}, c_{32}\right)=\left(1, \frac{1}{2}, \frac{1}{4}, \frac{1}{2}, \frac{1}{6}, \frac{2}{3}\right)
$$




\begin{tabular}{r|cccc}
$\Delta x[\mathrm{~km}] / k$ & 2 & 4 & 6 & 8 \\
\hline 2058 & & & $174 / 17920$ & $137 / 28800$ \\
1041 & $188 / 15360$ & $119 / 38400$ & $87 / 71680$ & $69 / 115200$ \\
522 & $94 / 61440$ & $60 / 153600$ & $44 / 286720$ & \\
261 & $47 / 245760$ & $30 / 614400$ & &
\end{tabular}

Table 1: Depending on the grid resolution $\Delta x[\mathrm{~km}]$ and the polynomial order $k$, the table contains ( model resolution $h[\mathrm{~km}] /$ number of grid unknowns ).

SSP methods combine high-order accuracy with stability properties respecting a CFL-condition. For nonlinear scalar conservation laws in one space-dimension [22] showed, that if the forward Euler method is total variation diminishing (TVD), then (8) is TVD with an appropriate CFL-condition, too. Although this does not prove stability for (8) applied to (7), it gives a good indication, that this SSP method is not a source of spurious oscillations in the discrete solution.

For a given polynomial degree $k$ in Sec. 3.2 a SSP method of order $k+1$ would be desirable. Only a third-order method (9) has been chosen, because all SSP-RK methods of higher order suffer from more restrictive CFL-conditions or require the construction of an adjoint operator. All numerical experiments in Sec. 4 show stable results even for $k>2$.

\section{Numerical Results}

The RK-DG method described in Sec. 3 has been used to implement a barotropic model of the atmosphere which has been validated performing numerical experiments. The validation process is carried out in two steps. At first, a convergence study considering steady-state and unsteady analytical solutions of the nonlinear SWE is performed. After that a barotropic instability in a localized jet stream has been carried out.

For the test with an analytical solution $q=\left(\Phi_{\text {ana }}, \Phi_{\text {ana }} u_{\text {ana }}\right)^{\mathrm{T}}$, the numerical error is evaluated using the normalized $L^{2}$-error of the geopotential field $\Phi_{h}$, that is

$$
\eta\left(\Phi_{h}\right)=\frac{\left\|\Phi_{\text {ana }}-\Phi_{h}\right\|_{L^{2}(S)}}{\left\|\Phi_{\text {ana }}\right\|_{L^{2}(S)}} .
$$

For every element $E$ a local grid resolution $\Delta x_{E}$, the size of the largest edge in $E$, and a local model resolution $h_{E}$ is defined by $h_{E}=\sqrt{|E| / N_{k}}$. Grid resolution $\Delta x$ and model resolution $h$ arise from the corresponding maximum values over all elements. Table 1 contains $h$ and the number of grid unknowns, depending on $\Delta x$ and the polynomial order $k$.

As a result of numerical experiments in every element $E$ the CFL-condition

$$
\lambda_{E} \frac{\Delta t_{E}}{h_{E}}=\frac{3}{4(k+1)}, \quad \Delta t \leq \Delta t_{C F L}=\min _{E \in \mathcal{T}} \Delta t_{E}
$$

was chosen where $\lambda_{E}$ is the characteristic wave speed $\lambda_{E}=|u|+\sqrt{\Phi}$. All numerical experiments gave stable results without spurious oscillations and a relaxed condition 


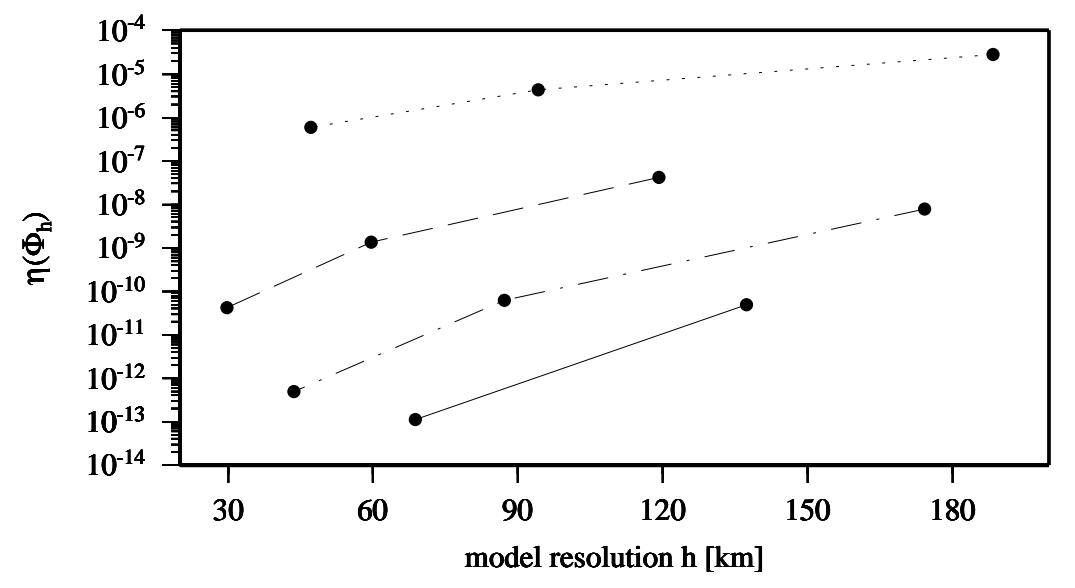

Figure 2: Section 4.1 (Steady-state Solid Body Rotation), Normalized $L^{2}$-error $\eta\left(\Phi_{h}\right)$ after integration time 5 days as a function of model resolution $h, k=2(\cdots), k=4$ $(--), k=6(-\cdot), k=8(-)$.

showed numerical instabilities. In the case $k=2$, with third order accuracy in space and time, this condition simplifies to

$$
\lambda_{E} \frac{\Delta t_{E}}{h_{E}}=\frac{1}{4}
$$

which is similar to the CFL-condition for linear stability in [5] in the one-dimensional scalar case. The critical time step length $\Delta t_{C F L}$ is derived in every time step, for given values $h_{E}$ and $\lambda_{E}$. All numerical experiments, except Sec. 4.2, have been performed with the time step $\Delta t=\Delta t_{C F L}$. Due to accuracy limitations the smaller time step $\Delta t=\frac{\Delta t_{C F L}}{4}$ is used for the experiments in Sec. 4.2. With this time step control the model shows stable numerical results and uses reasonable time step length.

\subsection{Steady-state Solid Body Rotation}

This test contains a steady-state solution of the nonlinear SWE, see [26, case 2]. The velocity field $u$ is a westerly wind with the meridional distribution of a solid body rotation. The geopotential height $\Phi$ is given in geostrophic balance to $u$. Thus, for the duration of the integration the initial data have to be maintained.

For the validation [26] recommend the evaluation of the normalized $L^{2}$-error $\eta\left(\Phi_{h}\right)$ after an integration time of 5 days. Fig. 2 shows $\eta\left(\Phi_{h}\right)$ for different polynomial orders $k=2,4,6,8$ as a function of the model resolution $h$. For all choices of the polynomial order $k$ the model converges and reduces the error almost up to machine precision for $k=6,8$. The errors decrease significantly for increasing $k$. Table 2 shows the expected order of convergence $k+1$. These results are very close to the convergence studies in [12], [20] and [11]. For this steady-state solution no limitation due to the third order RK method is observable. 


\begin{tabular}{r|cccc} 
Test $/ \mathrm{k}$ & 2 & 4 & 6 & 8 \\
\hline Section 4.1 & 2.86 & 4.97 & 6.97 & 8.78 \\
Section 4.2 & 3.02 & 5.02 & 4.83 & 4.20 \\
Section 4.3 & 4.84 & 5.70 & 7.25 & 8.79
\end{tabular}

Table 2: Sections 4.1, 4.2, 4.3, Experimental order of convergence for $k=2,4,6,8$.

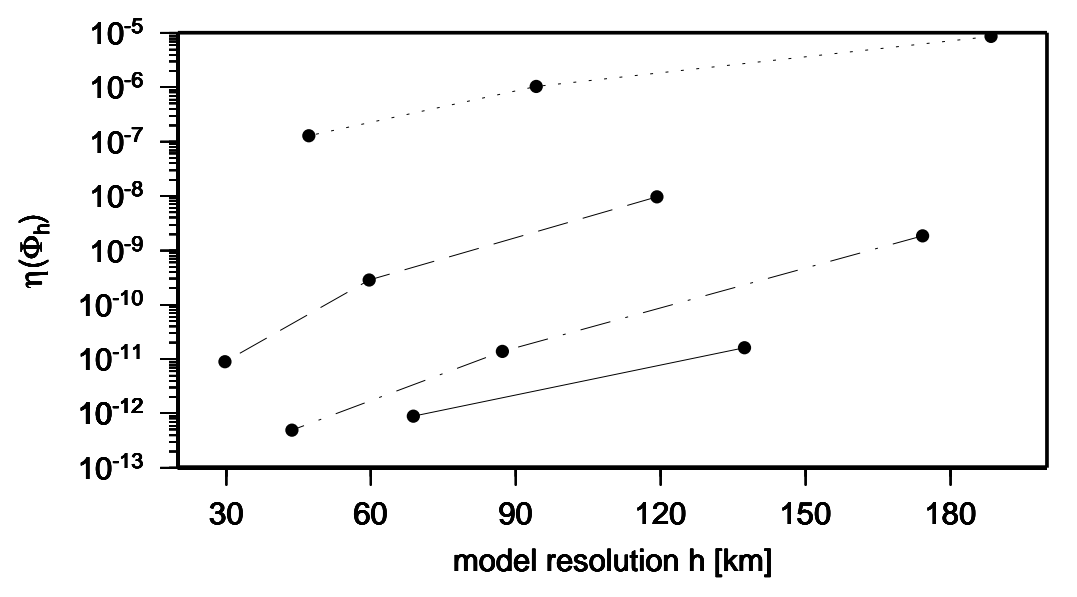

Figure 3: Section 4.2 (Unsteady Solid Body Rotation), Normalized $L^{2}$-error $\eta\left(\Phi_{h}\right)$ after integration time 5 days as a function of model resolution $h, k=2(\cdots), k=4$ $(--), k=6(-\cdot), k=8(-)$.

\subsection{Unsteady Solid Body Rotation}

This test concerns an unsteady solution of the nonlinear SWE, see [17, example 3]. Similar to the last test, the velocity field $u$ is a solid body rotation, but with an inclination to the Earth's rotation axis. An adequate unsteady geopotential field is available and an axially symmetric orographic field has to be regarded. This analytical solution moves into a westerly direction and has a time period of one day.

Again, the normalized $L^{2}$-error $\eta\left(\Phi_{h}\right)$ is evaluated after an integration time of 5 days. In this Section the time step is chosen as $\Delta t=\frac{\Delta t_{C F L}}{4}$, which is different from all other numerical experiments in this article. Fig. 3 shows $\eta\left(\Phi_{h}\right)$ for different polynomial orders $k=2,4,6,8$ as a function of the model resolution $h$. For all choices of the polynomial order $k$ the model converges, even in this unsteady test case. The errors decrease significantly for increasing $k$.

For all experiments with small errors $\left(\eta\left(\Phi_{h}\right) \leq 10^{-9}\right)$ the limiting factor for accuracy is the time step $\Delta t$ and not the model resolution $h$ any more. To see this, Fig. 4 is given, which shows $\eta\left(\Phi_{h}\right)$ as a function of $\Delta t$, for fixed parameters $(h, k)$. All experiments which are not plotted in Fig. 4 give the same results independent of the choice of $\Delta t \leq \Delta t_{C F L}$. It is to be seen, that $\eta\left(\Phi_{h}\right)$ stagnates for decreasing $h$, for fixed $\Delta t=\Delta t_{C F L}$ and all experiments with small errors. At the same time, for these experiments Fig. 4 shows $O\left(\Delta t^{3}\right)$ convergence of $\eta\left(\Phi_{h}\right)$. Both observations together yield, once $\eta\left(\Phi_{h}\right)$ is small enough, only a decreasing time step leads to 


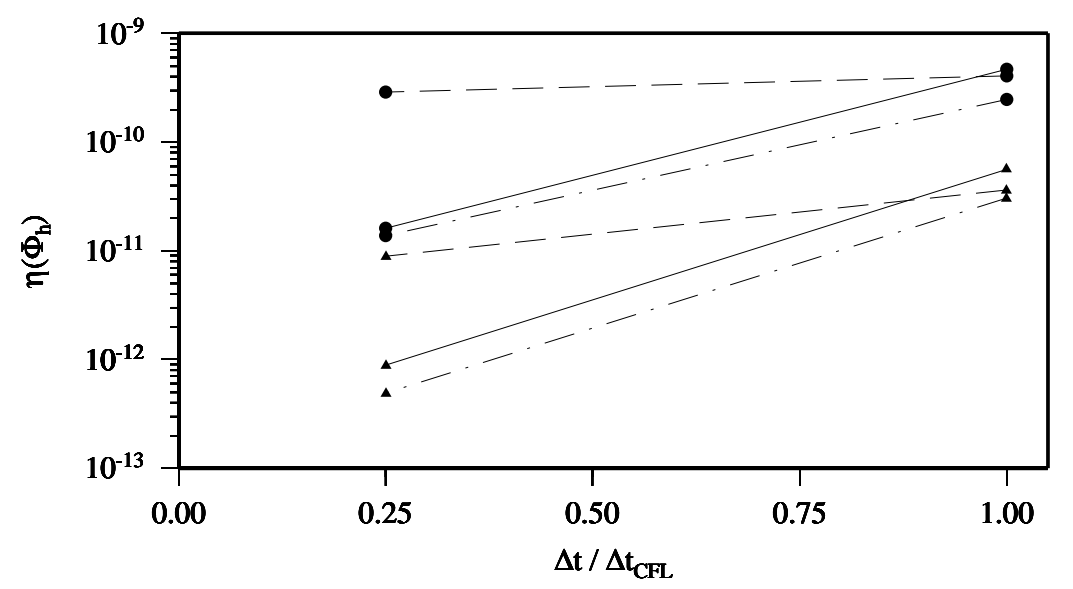

Figure 4: Section 4.2 (Unsteady Solid Body Rotation), Normalized $L^{2}$-error $\eta\left(\Phi_{h}\right)$ after integration time 5 days as a function of time step $\Delta t / \Delta t_{C F L},(h, k)=(60 \mathrm{~km}, 4)$ $(\bullet--),(h, k)=(30 \mathrm{~km}, 4)(\boldsymbol{\Delta}--),(h, k)=(87 \mathrm{~km}, 6)(\bullet-\cdot),(h, k)=(44 \mathrm{~km}, 6)$ $(\boldsymbol{\Delta}-\cdot),(h, k)=(137 \mathrm{~km}, 8)(\bullet-),(h, k)=(69 \mathrm{~km}, 8)(\boldsymbol{\Delta}-)$.

decreasing $\eta\left(\Phi_{h}\right)$. A further error reduction is anticipated for smaller time steps than $\frac{\Delta t_{C F L}}{4}$, which is not shown here due to limited computational resources. Of course this effect could be avoided using an appropriate RK method of order $k+1$. As explained in Sec. 3.4 a SSP-RK method with a tolerant CFL-condition is not available.

Table 2 shows for $k=2,4$ the expected order of convergence $k+1$. Because for $k=6,8$ the convergence is limited by the third order time step, in this case the convergence rates in space are suboptimal. At the same time the expected third order accuracy in time can be achieved. Because the absolute $L^{2}$-error $\left\|\Phi_{\text {ana }}\right\|_{L^{2}(S)}$ is larger compared to the similar steady-state case in Sec. 4.1, for the polynomial orders $k=2,4,6$ the normalized error is even smaller. The error for $k=8$ is limited by the time step, which results in slightly larger errors compared to Sec. 4.1.

\subsection{Unsteady Jet Stream}

This test contains a second unsteady solution of the nonlinear SWE, see [17, example 4]. This time, the velocity field $u$ is an axially symmetric westerly wind jet stream superimposed by a smaller solid body rotation, where the axis of the jet stream is inclined to the Earth's rotation axis. Due to the jet stream strong meridional gradients are present, which presents an additional difficulty compared to Sec. 4.2. As in Sec. 4.2, an adequate unsteady geopotential field is available and an axially symmetric orographic field has to be regarded. The solution moves into westerly direction and has a time period of one day.

The normalized $L^{2}$-error $\eta\left(\Phi_{h}\right)$ is evaluated after an integration time of 5 days. Fig. 5 shows the normalized $L^{2}$-error $\eta\left(\Phi_{h}\right)$ for different polynomial orders $k=$ $2,4,6,8$ as a function of the model resolution $h$. The method shows experimental convergence for this test case with strong meridional gradients. For increasing poly- 


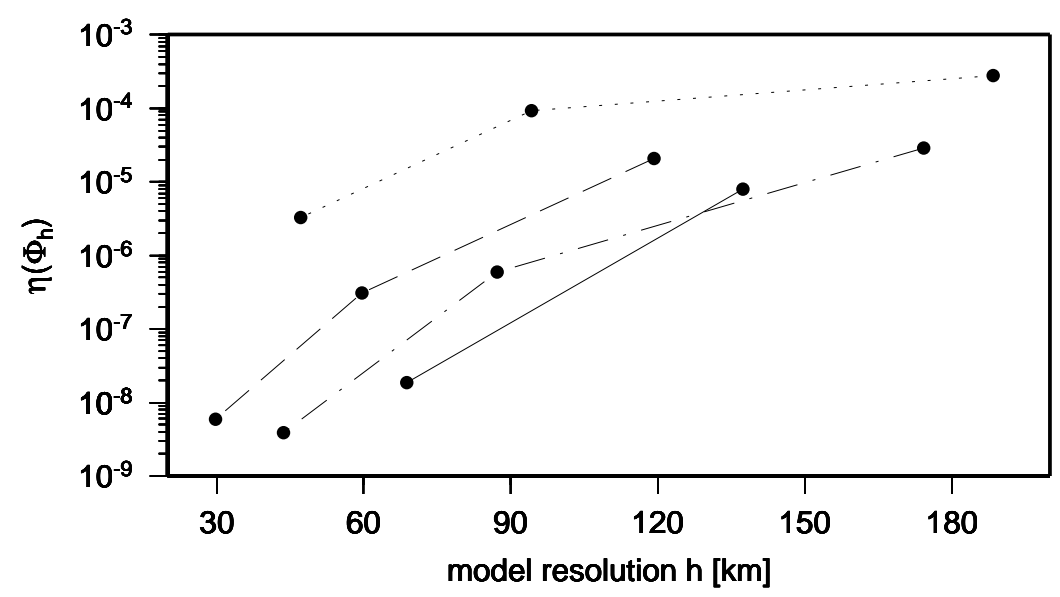

Figure 5: Section 4.3 (Unsteady Jet Stream), Normalized $L^{2}$-error $\eta\left(\Phi_{h}\right)$ after integration time 5 days as a function of model resolution $h, k=2(\cdots), k=4(--)$, $k=6(-\cdot), k=8(-)$.

nomial order $k$ the error $\eta\left(\Phi_{h}\right)$ decreases, but remains significantly larger than in Sec. 4.2. This is probably the reason for the order of convergence $k+1$, see Table 2 , without any limitation due to the third order RK method in this unsteady solution. Due to the locality of the jet stream, the 9 th-order experiment $(k=8)$ on a coarser grid resolves this test not as well as the 7th-order $(k=6)$ experiment which explains the crossing of both error lines.

\subsection{Perturbed Jet Stream}

Supplementing the standard tests for atmospheric models based on SWE, [9] proposed a test describing a barotropic instability. The initial velocity field $u$ is an axially symmetric westerly jet stream with the same axis as the Earth's rotation axis. As in Sec. 4.3, this jet stream includes strong meridional gradients and constitutes a rather local feature. Based on $u$ a geopotential height $\Phi$ is derived in geostrophic balance to $u$. Additionally a small perturbation $\Phi_{d}$ is added, such that the initial condition for the test is the geopotential field $\Phi+\Phi_{d}$. As a consequence of the perturbation $\Phi_{d}$, this experiment should not maintain the initial data. [9] give a detailed description of the barotropic instability developing within the jet stream from day four to day six.

At first, the model should be able to maintain the geostrophic balanced flow, as long as the initial perturbation does not lead to instabilities. Unlike for spectral models, this is not trivial for a model with a grid which is not aligned to the zonal flow. Fig. 6 shows the vorticity field after four days in a cutout of the global model domain for $k=2$ and two different model resolutions. Whereas a pronounced zonal wavenumber five is visible for $h=94 \mathrm{~km}$ the experiment with the higher resolution $h=47 \mathrm{~km}$ reduces the grid effect considerably. The same plot after four days is given in the upper plot of Fig. 7 for the model resoltuion $h=87 \mathrm{~km}$ but polynomial order $k=6$. This "high order" experiment seems to be more adequate to maintain the 

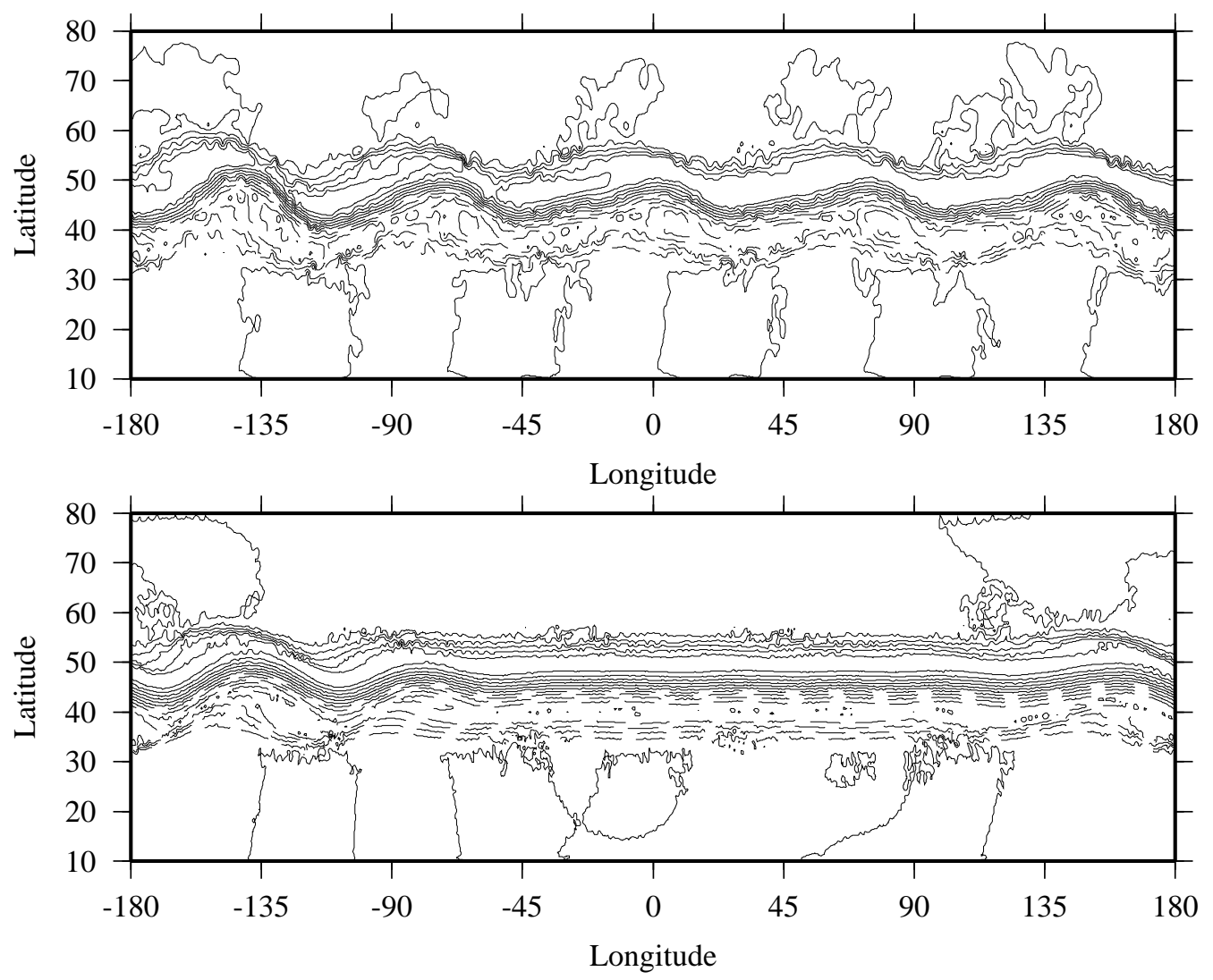

Figure 6: Section 4.4 (Perturbed Jet Stream), Vorticity (contour interval $2 \times$ $10^{-5} s^{-1}$, day $4, k=2$, top: $h=94 \mathrm{~km}$, bottom: $h=47 \mathrm{~km}$. 

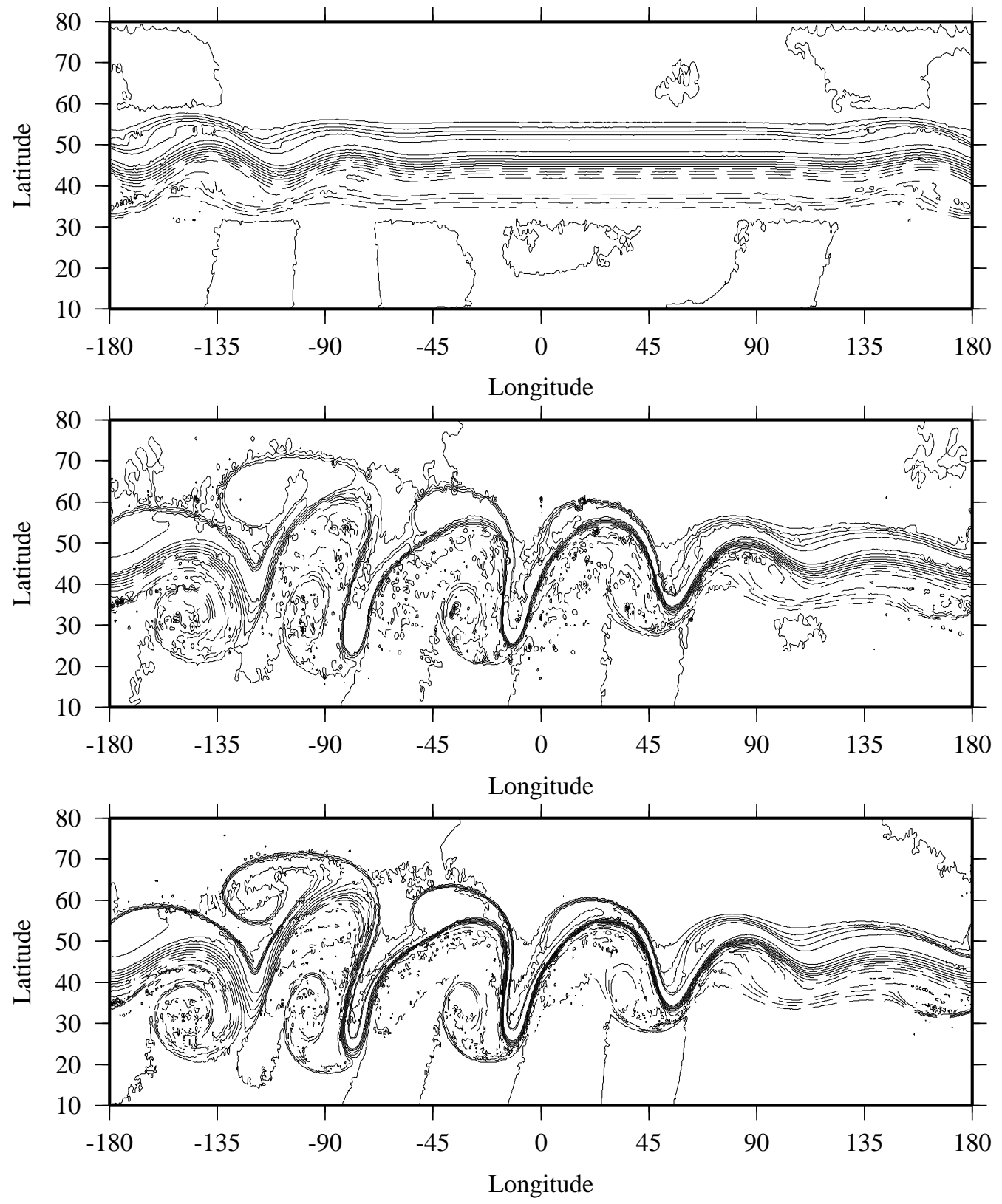

Figure 7: Section 4.4 (Perturbed Jet Stream), Vorticity (contour interval $2 \times$ $\left.10^{-5} s^{-1}\right), k=6$, top: day $4, h=87 \mathrm{~km}$, middle: day $6, h=87 \mathrm{~km}$, bottom: day $6, h=44 \mathrm{~km}$. 

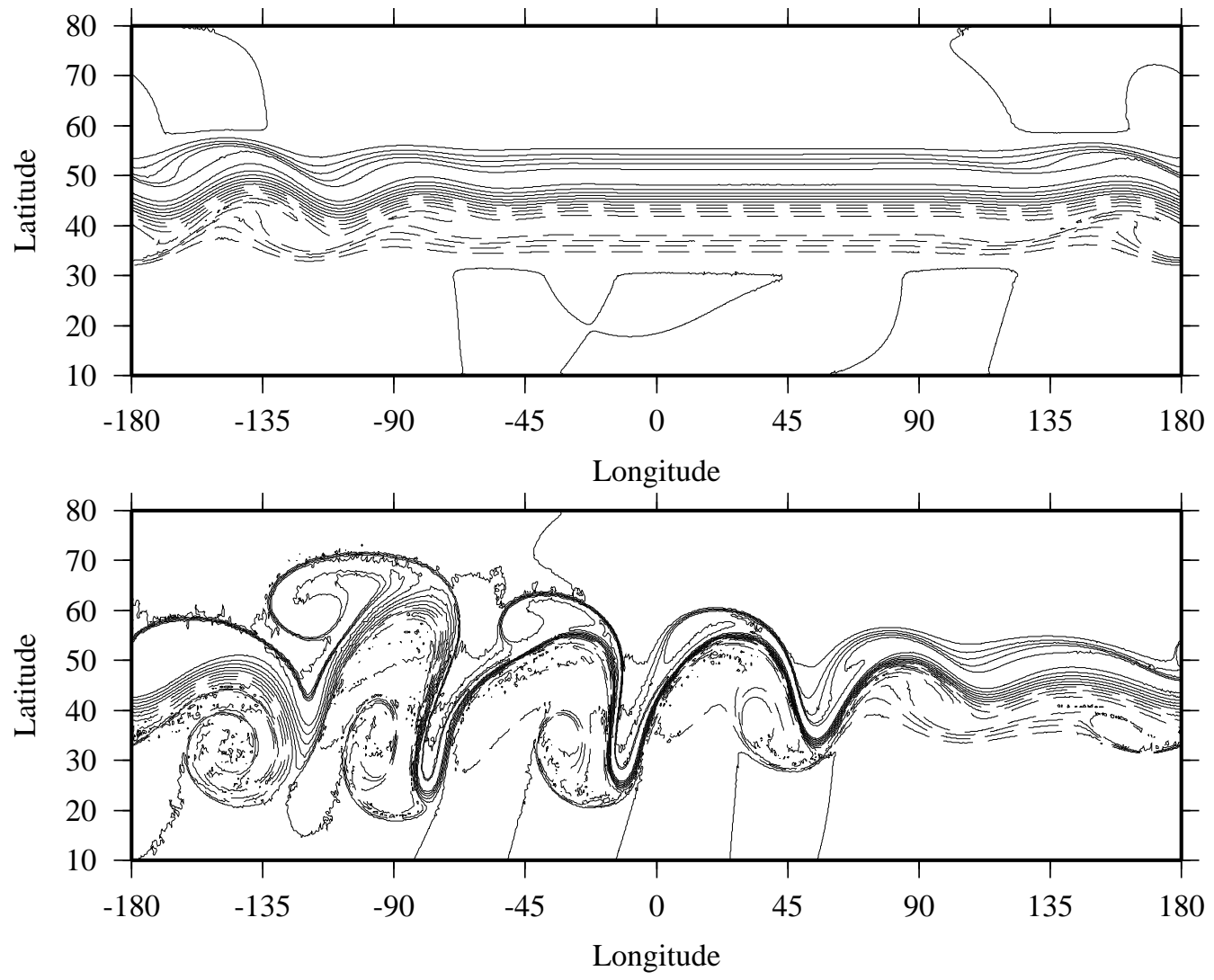

Figure 8: Section 4.4 (Perturbed Jet Stream), Vorticity (contour interval $2 \times$ $\left.10^{-5} s^{-1}\right),(h, k)=(30 \mathrm{~km}, 4)$, top: day 4 , bottom: day 6 .

geostrophic structure of the flow avoiding a low frequency zonal wave. The two lower plots of Fig. 7 show the vorticity for $k=6$ and the resolutions $87 \mathrm{~km}$ and $44 \mathrm{~km}$ after six days. In both experiments the laminar flow structure looks very similar, which gives an indication for the experimental convergence in smooth regions. In contrast to this, the areas with strong gradients, where barotropic instabilities develop, show spurious oscillations. The same experiment has been performed with polynomial order $k=4$ and high model resolution $h=30 \mathrm{~km}$, see Fig. 8 . Compared to the "low" and "high" order experiments in Figs. 6 and 7 the geostrophic balanced flow is maintained as well as the barotropic instabilities show only small spurious oscillations. Fig. 8 shows very good agreement with the inviscid run of [9] and the results in [23].

All numerical experiments have shown the discrete global conservation of mass up to machine precision, which is a direct consequence of the conservation properties of the DG method. The discrete global conservation of energy $E_{h}=\Phi_{h} u_{h}^{2}+\left(\Phi_{h}+\right.$ $\left.\Phi_{B}\right)^{2}-\Phi_{B}^{2}$ cannot be anticipated, because $E_{h}$ is not a conserved variable of the hyperbolic system (1). To investigate the discrete energy conservation, Fig. 9 shows the absolute value of the normalized energy error

$$
\eta\left(E_{h}\right)=\frac{\int_{S}\left(E_{h}-E_{a n a}\right) d x}{\left\|E_{\text {ana }}\right\|_{L^{1}(S)}}
$$




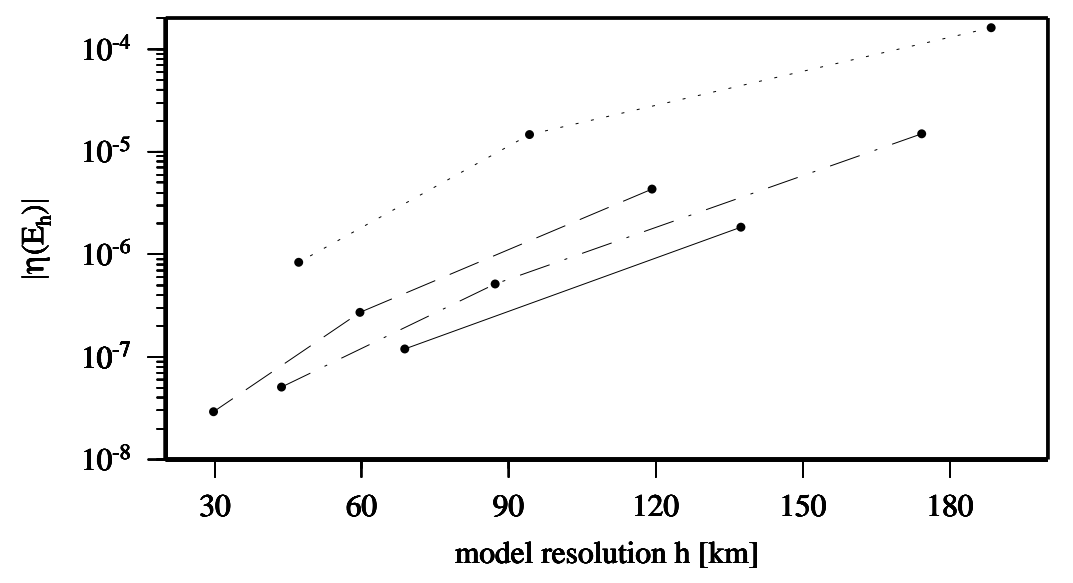

Figure 9: Section 4.4 (Perturbed Jet Stream), normalized energy error $\left|\eta\left(E_{h}\right)\right|$ as a function of model resolution $h, k=2(\cdot \cdot), k=4(--), k=6(-\cdot), k=8(-)$.

as a function of the model resolution $h$. The energy conservation is better than $2.0 \times 10^{-4}$, even for the coarse model resolution of $180 \mathrm{~km}$. Furthermore, for fixed polynomial orders $k$ and decreasing $h$ the energy error convergences. In the same manner for a fixed grid resolution $\Delta x$, an increasing $k$ leads to an energy error convergence. The time evolution of $\eta\left(E_{h}\right)$ is plotted in Fig. 10. For each $k$ only the experiment with the smallest model resolution $h$ is shown, see Table 1. For almost all experiments an energy loss is observed. For $k=2$ the error is much larger than for $k \geq 4$. This could be an indication for a loss of accuracy due to the $2 k-1$-order quadrature rule along the element edges. As long as the geostrophic flow remains stable, up to day four, the energy error remains very small. After the barotropic instability develops, after day four, all experiments show an enforced loss of energy. To explain this phenomenon we find, that the flow in the instability regions tends to develop discontinuities, which results in bigger jumps in the discrete solution. Considering this, [4] describes for a linear example, that the rate of dissipation in the DG method is given by the jumps of the solution. This property gives an explanation for the stronger energy loss during the development of barotropic instabilities.

\section{$5 \quad$ Summary and Outlook}

A global barotropic model of the atmosphere has been developed based on the spherical shallow water equations and discretized by a Runge-Kutta discontinuous Galerkin method. The equations are formulated as a hyperbolic conservation law on the sphere, a two-dimensional surface in $\mathbb{R}^{3}$, using coordinate independent differential opertators.

Spherical triangular coordinates, which are local coordinate mappings $\gamma_{E}$, and high-order polynomial spaces are defined on each curved element $E \subset S$ of a given spherical triangluar grid. $\gamma_{E}$ yields a two-dimensional representation of the tangential momentum fields and only two momentum equations which denotes a reduction of variables compared to the three-dimensional representation in a Cartesian coordi- 


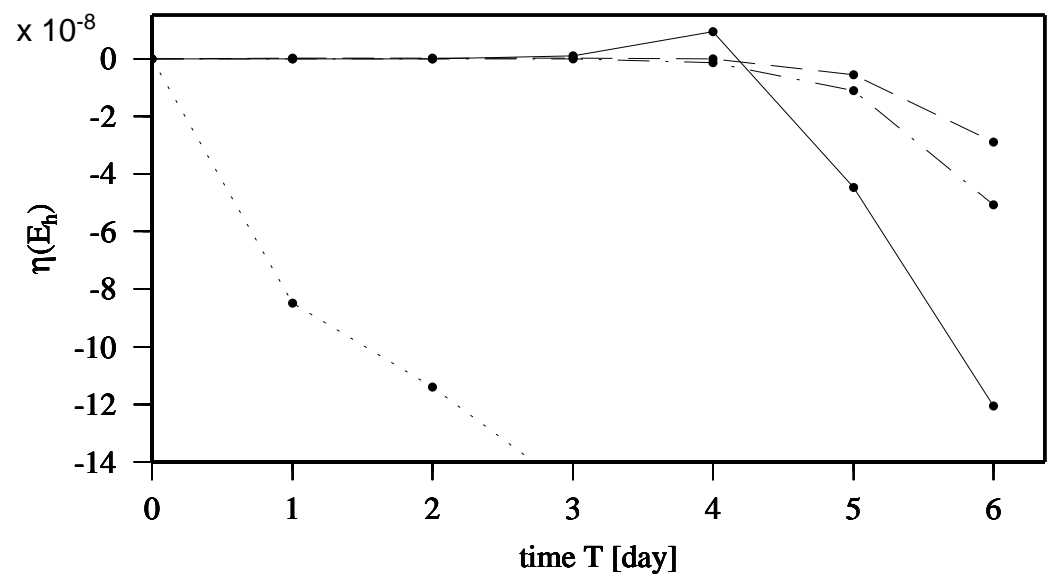

Figure 10: Section 4.4 (Perturbed Jet Stream), normalized energy error $\eta\left(E_{h}\right)$ as a function of integration time $T,(h, k)=(47 \mathrm{~km}, 2)(\cdots),(h, k)=(30 \mathrm{~km}, 4)(--)$, $(h, k)=(44 \mathrm{~km}, 6)(-\cdot),(h, k)=(69 \mathrm{~km}, 8)(-)$.

nate system. Governed by an integral form of the conservation law the space discrete discontinuous Galerkin method is obtained including a Rusanov numerical flux. An explicit third order Runge-Kutta method (strong stability-preserving) is applied to the space discrete system. The discrete method avoids any kind of explicit smoothing such as diffusion or filter operators. A barotropic model of the atmosphere is implemented based on the Runge-Kutta discontinuous Galerkin method completed by a time step control based on a CFL-condition. The model is validated regarding numerical experiments.

Steady-state and unsteady analytical solutions of the nonlinear shallow water equations are considered. In all cases the model achieves experimental convergence to the known solution. Furthermore, for increasing polynomial order $k$, the error decreases significantly. For the steady-state solid body rotation and the unsteady jet stream the experimental orders of convergence are $k+1$. This convergence rate is achieved for the unsteady solid body rotation, only if $k \leq 4$. For higher orders $k$ and very small errors the time step $\Delta t$ limits the error (third order Runge-Kutta method). This result of the presented atmospheric model demonstrates, that the model time step is restricted not only by a CFL-condition but by accuracy demands, too. Further, a qualitative test regarding a barotropic instability within a perturbed jet stream is performed. In very good agreement to published results the experiment shows the evolution of a breaking barotropic wave after six days without generating spurious oscillations. The global energy error converges with decreasing grid resolution as well as with increasing polynomial order. For all numerical experiments the anticipated mass conservation is achieved up to machine precision.

The presented results show the great potential of the Runge-Kutta discontinuous Galerkin method in a simplified atmospheric model. Thus, the application to three dimensional atmospheric equations, which form a hyperbolic system, seems to be warranted. Further, the method demonstrates the application of local coordinate mappings, here spherical triangular coordinates, and its capacity to represent tan- 
gential momentum fields that are locally two-dimensional. Using the same technique for global atmospheric models in three-dimensional prismatic grids yields the useful decomposition into tangential and vertical components.

\section{Acknowledgements}

The authors gratefully acknowledge the support of the Office of Naval Research Global through the Grant N00014-07-1-4038. The second author also gratefully acknowledges the support of the Office of Naval Research through PE-0602435N.

\section{Appendix, Manifolds}

The spherical domain $S$ is regarded as a two-dimensional $C^{1}$-manifold embedded in $\mathbb{R}^{3}$. Thus, the operators and Gauß' theorem on $S$ are special cases of the standard definitions from differential geometry. Because the local representation of the operators and the integrals regarding a coordinate mapping $\gamma$ are essential to the implementation of the model, these formulas are given below.

Let $f \in C^{1}(S)$ be a scalar function and $u \in C^{1}\left(S, \mathbb{R}^{3}\right)$ a vector field with $u(x) \in$ $T_{x}(S)$. Further, for a fixed $x \in S$ let $\left(\tau_{1}, \tau_{2}\right)$ be a orthonormal basis of $T_{x}(S)$. Then the spherical gradient and spherical divergence are defined by

$$
\nabla_{S} f(x)=\sum_{i=1}^{2} \partial_{\tau_{i}} f(x) \tau_{i}, \quad \operatorname{div}_{S} u(x)=\sum_{i=1}^{2} \tau_{i} \cdot \partial_{\tau_{i}} u(x) .
$$

Then, for an open subset $E \subset S$ with smooth boundary a formulation of Gauß' theorem on $S$ is

$$
\int_{E} f \operatorname{div}_{S} u d x=-\int_{E} u \cdot \nabla_{S} f d x+\int_{\partial E} f u \cdot \nu_{E} d \sigma .
$$

Now, let $\Omega \subset S$ be open with a local coordinate mapping $\gamma: \tilde{\Omega} \subset \mathbb{R}^{2} \rightarrow \Omega$, $D \subset \tilde{\Omega}, \bar{E} \subset \Omega$ and $\gamma(D)=E$. Further, remember $\left(b^{1}, b^{2}\right)$ the dual basis of $T_{x}(S)$ and Gram's determinant $g$.

Let $D \subset \mathbb{R}^{2}$ open, $E \subset S$ open in $S$ and $\gamma: D \rightarrow E$ a local coordinate mapping. $\gamma$ generates the local coordinate system

$$
b_{i}=\partial_{y_{i}} \gamma, \quad i=1,2
$$

which is a basis of the tangential space $T_{x}(S)$. The condition $b_{i} \cdot b^{j}=\delta_{i}^{j}$ determines the dual basis $\left(b^{1}, b^{2}\right)$. Gram's matrix (metric tensor), its inverse and Gram's determinant are defined by

$$
g_{i j}=b_{i} \cdot b_{j}, \quad g^{i j}=b^{i} \cdot b^{j}, \quad g=\operatorname{det}\left(g_{i j}\right)_{i, j=1,2} .
$$

Derivatives of the basis functions $b_{i}$ are expressed using Christoffel symbols which fulfill the equations

$$
\partial_{y_{j}} b^{i}=-\Gamma_{j k}^{i} b^{k}, \quad \Gamma_{i j}^{k}=\frac{g^{n k}}{2}\left(\partial_{y_{i}} g_{j n}+\partial_{y_{j}} g_{i n}-\partial_{y_{n}} g_{i j}\right)
$$


Then, for $y \in D$ the operators fulfill the local representations

$$
\left.\nabla_{S} f\right|_{\gamma(y)}=\sum_{i=1}^{2}\left[\partial_{y_{i}}(f \circ \gamma)\right]_{y} b^{i},\left.\quad \operatorname{div}_{S} u\right|_{\gamma(y)}=\sum_{i=1}^{2} \frac{1}{\sqrt{g}}\left[\partial_{y_{i}} \sqrt{g}(u \circ \gamma) \cdot b^{i}\right]_{y} .
$$

The face and line integrals satisfy the local representations

$$
\int_{E} f d x=\int_{D} \sqrt{g} f \circ \gamma d y, \quad \int_{\partial E} f d \sigma=\int_{\partial D} \sqrt{g}\left|\left(b^{1}, b^{2}\right) \cdot \nu_{D}\right| f \circ \gamma d \sigma
$$

where $\nu_{D}$ is the unit normal on $\partial D$.

\section{References}

[1] J. Behrens, N. Rakowsky, W. Hiller, D. Handorf, M. Läuter, J. Päpke, and K. Dethloff. amatos: parallel adaptive mesh generator for atmospheric and oceanic simulation. Ocean Modelling, 10:171-183, 2005.

[2] L. Bos, M. A. Taylor, and B. A. Wingate. Tensor product Gauss-Lobatto points are Fekete points for the cube. Mathematics of Computation, 70:1543-1547, 2001.

[3] P. G. Ciarlet and P.-A. Raviart. Interpolation theory over curved elements, with applications to finite element methods. Comput. Methods Appl. Mech. Engrg., 1:217-249, 1972.

[4] B. Cockburn. Discontinuous Galerkin methods. ZAMM Z. Angew. Math. Mech., 83:731-754, 2003.

[5] B. Cockburn and C.-W. Shu. Runge-Kutta discontinuous Galerkin methods for convection-dominated problems. J. Sci. Comput., 16:173-261, 2001.

[6] R. Cools. Monomial cubature rules since Stroud: a compilation - part 2. J. Comput. Appl. Math., 112:21-27, 1999.

[7] R. Cools and P. Rabinowitz. Monomial cubature rules since Stroud: a compilation. J. Comput. Appl. Math., 48:309-326, 1993.

[8] J. Côté. A Lagrange multiplier approach for the metric terms of semiLagrangian models on the sphere. Q. J. R. Meteorol. Soc., 114:1347-1352, 1988.

[9] J. Galewsky, R.K. Scott, and L. M. Polvani. An initial-value problem for testing numerical models of the global shallow water equations. Tellus A, 56:429-440, 2004.

[10] F. X. Giraldo. A spectral element shallow water model on spherical geodesic grids. Int. J. Numer. Meth. Fluids, 35:869-901, 2001. 
[11] F. X. Giraldo. High-order triangle-based discontinuous Galerkin methods for hyperbolic equations on a rotating sphere. J. Comput. Phys., 214:447-465, 2006.

[12] F. X. Giraldo, J. S. Hesthaven, and T. Warburton. Nodal high-order discontinuous Galerkin methods for the spherical shallow water equations. J. Comput. Phys., 181:499-525, 2002.

[13] F. X. Giraldo and T. Warburton. A nodal triangle-based spectral element method for the shallow water equations on the sphere. J. Comput. Phys., 207:129-150, 2005.

[14] S. Gottlieb, C.-W. Shu, and E. Tadmor. Strong stability-preserving high-order time discretization methods. SIAM Review, 43:89-112, 2001.

[15] J. S. Hesthaven. From electrostatics to almost optimal nodal sets for polynomial interpolation in a simplex. SIAM J. Numer. Anal., 35:655-676, 1998.

[16] L. Krivodonova, J. Xin, J.-F. Remacle, N. Chevaugeon, and J. E. Flaherty. Shock detection and limiting with discontinuous Galerkin methods for hyperbolic conservation laws. Appl. Numer. Math., 48:323-338, 2004.

[17] M. Läuter, D. Handorf, and K. Dethloff. Unsteady analytical solutions of the spherical shallow water equations. J. Comput. Phys., 210:535-553, 2005.

[18] S.-J. Lin and R. B. Rood. An explicit flux-form semi-Lagrangian shallow-water model on the sphere. Q. J. R. Meteorol. Soc., 123:2477-2498, 1997.

[19] J. Lyness and R. Cools. A survey of numerical cubature over triangles. Proc. of Symp. in Appl. Math., 48:127-150, 1994.

[20] R. D. Nair, S. J. Thomas, and R. D. Loft. A discontinuous Galerkin global shallow water model. Mon. Wea. Rev., 133:876-888, 2005.

[21] J. A. Rossmanith. A wave propagation method for hyperbolic systems on the sphere. J. Comput. Phys., 213:629-658, 2006.

[22] C.-W. Shu and S. Osher. Efficient implementation of essentially non-oscillatory shock-capturing schemes. J. Comput. Phys., 77:439-471, 1988.

[23] A. St-Cyr, C. Jablonowski, J. M. Dennis, H. M. Tufo, and S. J. Thomas. A comparison of two shallow water models with non-conforming adaptive grids. Mon. Wea. Rev., 2007. accepted for publication.

[24] A. H. Stroud. Approximate Calculation of Multiple Integrals. Prentice-Hall, London, 1971.

[25] M. Taylor, J. Tribbia, and M. Iskandarani. The spectral element method for the shallow water equations on the sphere. J. Comput. Phys., 130:92-108, 1997. 
[26] D. L. Williamson, J. B. Drake, J. J. Hack, R. Jakob, and P. N. Swarztrauber. A standard test set for numerical approximations to the shallow water equations in spherical geometry. J. Comput. Phys., 102:211-224, 1992. 